\title{
Ablation vs. amiodarone for treatment of persistent atrial fibrillation in patients with congestive heart failure and an implanted device
}

\author{
Djouhar Roufeida Belgaid* \\ Barts and the London School of Medicine and Dentistry, London, UK
}

\begin{abstract}
Objectives: Assess whether catheter ablation is superior to amiodarone for the treatment of persistent atrial fibrillation (AF) in patients with heart failure as well. Methods: This was a randomised multicenter study. Patients selected for the trail had the following within the last 6 month:

1. Persistant AF

2. Dual chamber implantable cardioverter device or Cardiac Resynchronization Therapy for Defibrillators

3. NYHA class II-III

4. Left ventricular ejection fraction $<40 \%$

They were then randomly assigned at a 1:1 ratio to group 1- undergo catheter ablation (102 participants) or group 2- receive amiodarone (101 participants)

Main outcome measures: Primary end-point was the recurrence of AF. Secondary endpoints were all-cause mortality and hospitalization. Patients were then followed up for at least 24 months.

Results: $71(70 \%$ [95\% CI 60\% - 78\%]) patients in the catheter ablation group did not experience a recurrence after $1.4 \pm 0.6$ procedures compared to 34 (34\% [95\% CI 25\% - 44\%]). Moreover, the rate of success after the first ablation was between 29 to $61 \%$. The rate of emergency hospitalization during the follow up was $31 \%$ in the ablation group and $57 \%$ in the amiodarone group. Lastly, the rate of mortality in the catheter ablation group was $8 \%$ compared to $18 \%$ in the amiodarone group.

Conclusion: Catheter ablation is found to be superior to amiodarone in achieving freedom from recurrent atrial fibrillation at the follow up. There was also a reduction in mortality and unplanned hospitalisation in patients with persistent atrial fibrillation and heart failure [1].
\end{abstract}

\section{Background information and rationale for carrying out work}

Catheter ablation is a minimally invasive intervention used as an alternative when long term drugs prove to be ineffective or contraindicated in patients with persistent atrial fibrillation. It is a reasonable alternative to the pharmacological cardioversion such as amiodarone in an attempt to restore a normal cardiac rhythm [2].

Based on previous trials, one of the main advantages of using catheter ablation over anti-arrhythmic drugs is its localised action therefore avoids systemic side effects (e.g.: dizziness, diarrhoea, pulmonary fibrosis) seen with the drugs [3]. The reason why performing this trial was beneficial is because previous catheter ablation trials conducted utilised patients with preserved left ventricular function. Results from these trials consistently showed that it has been successful in improving the quality of life of patients [4] and improving morbidity [5].

Due to the tachycardia, induced myopathy and remodelling of the atria, heart failure predisposes patients to atrial fibrillation simultaneously atrial fibrillation exacerbates heart failure. Thus, these two conditions often coincide, with most patients also having a left ventricular systolic dysfunction. Moreover, the occurrence atrial fibrillation in patients with heart failure (HF) as well increases from $<10 \%$ in those with NYHA class I of HF to $50 \%$ in those with NYHA class IV of HF. This further highlights the importance of performing trials to improve symptoms and reduce mortality in patients with atrial fibrillation with a background of heart failure and low ejection systolic fraction as it is becoming increasingly common [6]. The aim of this randomised control trial (RCT) is to assess if catheter ablation is superior to amiodarone, an antiarrhythmic drug, in patients with persistent atrial fibrillation and heart failure.

\section{Approaches to the question and key results}

\section{Study design}

AATAC is a RCT which aims to determine if catheter ablation is

Correspondence to: Djouhar Roufeida Belgaid, Barts and the London School of Medicine and Dentistry, London, UK, E-mail: d.r.belgaid@smd13.qmul.ac.uk

Key words: atrial fibrillation, heart failure, catheter ablation, arrhythmia, amiodarone

Received: October 20, 2016; Accepted: November 09, 2016; Published: November 12, 2016 
better than amiodarone to treat persistent atrial fibrillation. I think that this trial addressed the objective well, as demonstrated through the study design. Patients taking part in this trial had to be 18 years or over with all the following within the last 6 months:

1. Persistent atrial fibrillation

2. Dual chamber ICD or CRTD

3. NYHA stage II-III of heart failure

4. Left ventricular ejection fraction

However, patients with any of the conditions listed in Table 1 were excluded from the trial as it might interfere with the results due to confounding factors. In addition, factors adjusted in the multivariable model included age, gender, hypertension and diabetes. This helps improve the reliability of the results. After gaining consent from eligible patients, they were randomly assigned to group 1 (102 participants) receive catheter ablation or group 2 (101 participants) amiodarone. The first 3 months of the trial was when ablation was performed and amiodarone was titres. Data was not collected during this period. Trial period continued for 21 months after (total duration 24 months).

\section{Endpoints}

The primary endpoint for this study is the long-term procedural success. This includes lack of recurrence of atrial fibrillation/ flutter/ tachycardia for more than 30 seconds off the antiarrhythmic drugs at the follow up. In the ablation group, a second ablation can be performed with in the first three months' period (blanking period). However, after this period any atrial arrhythmia is categorised as a recurrence.

The secondary endpoints included the following:

1. All-cause mortality

2. Atrial fibrillation and heart failure related emergency hospitalisation during the post ablation follow up period

3. Changes in the left ventricular ejection fraction

4. 6-minute walk distance (6MWD)

5. Quality of life assessed by Minnesota Living with Heart Failure Questionnaire (MLHFQ)

\section{Methods}

For the ablation group, dofetilide was stopped around 5 days before the ablation or if patients were on low dose amiodarone (less than $200 \mathrm{mg}$ ), they stopped taking it after the first three months (blanking period). Perhaps this might have affected the results as haven't been adjusted for this factor. Double Tran septal was performed, IV heparin administered and circular mapping catheter used to guide the ablation.

Table 1. List of the exclusion criteria for the trial.

\begin{tabular}{|l|}
\hline Exclusion Criteria \\
\hline Atrial fibrillation caused by reversible etiology \\
\hline Valvular disease requiring surgical intervention \\
\hline Coronary heart disease requiring surgical intervention \\
\hline Early post-operative atrial fibrillation (within 3 months of surgery) \\
\hline Life expectancy of 2 years or less \\
\hline Prolonged QT interval \\
\hline Hypothyroidism \\
\hline History of severe pulmonary disease \\
\hline Liver failure \\
\hline Receiving regular dose amiodarone each day \\
\hline
\end{tabular}

The main aim of the ablation procedure was pulmonary vein antrum (PVI) \pm left atrial posterior wall isolation.

For the amiodarone group, a loading dose of $400 \mathrm{mg}$ twice a day was given for 2 weeks then $400 \mathrm{mg}$ each day for the two weeks after. A maintenance dose $200 \mathrm{mg}$ a day was then started. 12 patients in the ablation group and 15 in the amiodarone group receiving low dose amiodarone i.e., $<200 \mathrm{mg} /$ day were included in the trial. This, as mentioned previously, might have affected the reliability of the results due to the confounding effect.

With regards to screening, pulmonary function tests and chest $\mathrm{x}$-ray was performed at baseline then yearly, as well as liver function tests performed at baseline then every 6 months. 2D and Doppler echocardiography was also performed to quantify the left ventricular ejection fraction of both group. ECG, NYHA class, ECHO, MLHFQ and 6MWD were all monitored at the start for baseline reading and at the 24 month follow up. Moreover, as all patients have chronic heart failure they were all receiving the optimal medical therapy.

\section{Results}

In the post blanking period (4-24 months) 15 patients in the ablation group and 25 patients in the amiodarone group received cardioversion. At the end of the trial, 71(70\% [95\% CI 60\%-78\%]) patients in the catheter ablation group did not experience a recurrence after $1.4 \pm 0.6$ procedures compared to 34 ( $34 \%$ [95\% CI $25 \%-44 \%]$ ) in the amiodarone group. Of the remaining 67 patients who had an arrhythmia recurrence in the amiodarone group, 7 of them failed after experiencing side effects (thyroid toxicity, pulmonary toxicity and liver dysfunction).

In the ablation group with 102 patients, PVI with posterior wall isolation was performed on 80 but only PVI was performed on the rest of the 22 patients. 63 patients (79\% 95\%CI 68-86\%]) who underwent PVI as well as posterior wall isolation reported freedom from recurrence. This is in contrast to only 8 patients (36\% [95\% CI 17\%-56\%]) that only had PVI. This shows that posterior vein isolation with posterior wall isolation is more successful. Moreover, the rate of success after the first ablation was between 29 to $61 \%$.

Results as summarized in Table 2 from the unadjusted Cox model shows that amiodarone treatment and diabetes mellitus has the highest association with the recurrence of arrhythmia. After adjusting the results to take into consideration the confounding factors mentioned in Table 2, patients receiving amiodarone had the greatest failure rate. Their hazard ratio was 2.5 [ $95 \%$ CI 1.5 to 4.3 ], p $<0.001$, when compared to the catheter ablation group.

The rate of emergency hospitalization during the follow up was lower in the ablation group (32 participants) compared to the amiodarone group (58 participants). Thus, the relative risk reduction is $45 \%$.

Table 2. Univariate association with the recurrence of arrhythmia.

\begin{tabular}{|l|c|c|}
\hline Variables & Hazard Ratio & $\mathbf{9 5 \%}$ Confidence Limits \\
\hline Amiodarone Treatment & 3.00 & 1.96 to 4.61 \\
\hline Sex & 1.14 & 0.92 to 1.41 \\
\hline Age, years & 0.99 & 0.98 to 1.02 \\
\hline Body Mass Index & 0.99 & 0.94 to 1.03 \\
\hline LVEF, \% & 0.96 & 0.93 to 0.99 \\
\hline Hypertension & 1.12 & 0.93 to 1.36 \\
\hline Left Atrial Size & 1.02 & 0.99 to 1.05 \\
\hline Diabetes Mellitus & 2.22 & 1.31 to 3.75 \\
\hline
\end{tabular}


Lastly, the rate of mortality was also lower in the catheter ablation group (8 participants) compared to the amiodarone group (18 participants). Thus, the relative risk reduction is $56 \%$.

\section{Likely impact of research outcome}

Atrial fibrillation and heart failure are very common cardiac conditions and often coexist. This study provides valuable data to demonstrate that catheter ablation is in fact superior to the use of amiodarone to achieve freedom from atrial fibrillation recurrence in patients with atrial fibrillation and heart failure. It is important to note that this is the first randomised control trail that provides these results and demonstrates that ablation reduces unplanned hospitalisation, mortality and improved the exercise capacity of patients and their quality of life. This clearly shows the advantages and clinical relevance of using ablation over amiodarone in patients with heart failure as patients have an improved wellbeing and use of health resources has reduced

As per the recommendations of the international guidelines, patients with atrial fibrillation with a background of heart failure should be on sinus rhythm control drugs like amiodarone and dofetilide [7]. As they are oral drugs, they come with unwanted systemic side effects. Amiodarone can lead to pulmonary fibrosis, hepatic/thyroid toxicity, bradycardia and corneal deposits. Similarly, dofetilide can cause QT prolongation, torsade de points, dizziness and confusion. Therefore, as ablation therapy is a localised intervention these adverse effects can be avoided and sinus rhythm restored. Moreover, the fact that ablation has helped improve symptoms, quality of life and left ventricular function regardless of the rate control before the intervention demonstrated that other factors are responsible for the worsening of cardiac function and not just the rate control [1]. In addition, factors that have contributed to the higher success rate of ablation in this study includes greater operator experience and enhancement in the mapping and ablation technologies. As the results demonstrated, an average of 1.4 ablation procedures was enough for patients to become free from arrhythmia recurrence. It is also important to note that having a PVI with posterior wall isolation proved to be significantly more successful than having PVI done alone.

\section{Future work}

Perhaps a longer study with more participants would be useful to monitor the long-term effectiveness of catheter ablation, in reducing mortality and hospitalisation as well as relieving symptoms. As the international guidelines, recommends the use of amiodarone or dofetilide for rhythm control of atrial fibrillation for patients with a background of heart failure; it would be interesting to see if the results would be similar if dofetilide would be used instead in the control group. Similarly, it would be interesting to test out the catheter ablation group against rate control drugs as opposed to rhythm to see if the results would be consistent. Bearing in mind that amiodarone has rate control properties. Patients who were on low dose $(<200 \mathrm{mg} / \mathrm{d})$ amiodarone where allowed to take part in the ablation group. This might have affected the reliability of the results. Perhaps those patients would have been also part of the exclusion criteria.

\section{Conclusion}

Catheter ablation is found to be superior to amiodarone in achieving freedom from recurrent atrial fibrillation at the follow up. Patients in the ablation group had an improved quality of life, symptoms and exercise tolerance. There was also a reduction in mortality and unplanned hospitalisation in patients with persistent atrial fibrillation and heart failure.

\section{Conflicts of interests}

None declared

\section{References}

1. Luigi Di Biase, Prasant Mohanty, Sanghamitra Mohanty, Pasquale Santangeli, Chintan Trivedi, et al. (2016) Ablation Versus Amiodarone for Treatment of Persistent Atria Fibrillation in Patients with Congestive Heart Failure and an Implanted device. Circulation 133: 1637-1644

2. Non-surgical Procedures for Atrial Fibrillation (AFib or AF) (2016) American Heart Association

3. Hutter S (2016) Pros and Cons of Medication vs. Ablation for Atrial Fibrillation EverydayHealth.com

4. Hsu L, Jais P, Sanders P (2005) Catheter ablation for atrial fibrillation in congestive heart failure. ACC Current Journal Review 14: 44.

5. Choi A, Hematpour K, Kukin M, Mittal S, Steinberg J (2010) Ablation vs Medical Therapy in the Setting of Symptomatic Atrial Fibrillation and Left Ventricular Dysfunction. Congestive Heart Failure 16: 10-14.

6. Maisel WH, Stevenson LW. (2003) Atrial fibrillation in heart failure: epidemiology, pathophysiology, and rationale for therapy. Am J Cardiol 91: 2-8. [Crossref]

7. Parikh PVenkatachalam K. (2016) Optimizing Heart Rate and Controlling Symptoms in Atrial Fibrillation. US Cardiology Review 10: 26.

Copyright: (C2016 Belgaid DR. This is an open-access article distributed under the terms of the Creative Commons Attribution License, which permits unrestricted use, distribution, and reproduction in any medium, provided the original author and source are credited. 